\title{
Bufoténine et bufoténidine : des alcaloïdes aux vertus hallucinogènes et aphrodisiaques extraits du venin de crapaud
}

\section{Bufotenine and bufotenidine : hallucinogenic and aphrodisiac alleged alcaloids extracted from toad venom}

Jean-Pierre ANGER*(1), Pascal KINTZ ${ }^{(2)}$

(1) Université de Rennes 1 - Rennes Cedex - France (2) Laboratoire ChemTox, Ilkirch-Graffenstaden - France

*Auteur à qui adresser la correspondance : Jean-Pierre ANGER, 10, allée de la Rivière - 35760 Saint Grégoire Tél : 0299689239

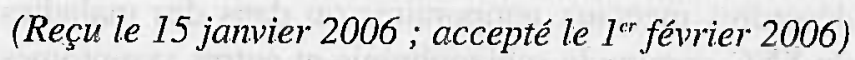

\section{RÉSUMÉ}

La bufoténine (5-hydroxy-N,N-diméthyltryptamine) est un alcaloïde indolique de la famille des tryptamines comme la sérotonine, la N-méthylsérotonine, la 5-méthoxy-N-méthyltryptamine et la mélatonine retrouvées dans les graines de certaines légumineuses ainsi que dans les sécrétions de la peau de différents amphibiens. Chez l'homme, ces molécules possèdent de puissantes propriétés psychotropes et sont impliquées dans les désordres mentaux temporaires ou dans des maladies du cerveau comme la schizophrénie et autres symptômes psychotiques, probablement du fait de leur point commun tant sur le plan structural que physiologique avec le L.S.D, vis à vis du récepteur 5-HT2 .

Le présent travail se propose de faire le point sur la composition et les propriétés du venin de crapaud, ses prétendues vertus hallucinogènes et aphrodisiaques, ce que l'on sait actuellement de ses effets sur l'homme et des risques d'intoxication.

Historiquement les crapauds Bufo ont été extrêmement. importants pour différentes cultures à travers le monde, durant des millénaires. Ils semblent aujourd'hui susceptibles

\section{SUMMARY}

Bufotenin (5-hydroxy-N,N-dimethyltryptamine) is a tryptamine alkaloid, such as serotonin, $N$-methylserotonin, 5methoxy- $N$-methylserotonin and melatonin, widely distributed in the leguminosae family and commonly found in a number of vertebrate as mammals and in many anphibian groups around the world. In human, these molecules show potent psychotropic properties an are usually associated to temporary mental disorders and brain deseases such as schizophrenia and other psychotic symptoms, probably due to their similar physiological and structural features to LSD in the $5 H T 2$ receptor:

The present study investigates the properties of toad venom, its hallucinogenic and aphrodisiac alleged properties, what we know about its psychedelic effects and the toxicological hazards for humans. Some papers report the use of toad venom by ingestion or by sniffing in the United States and in Australia. This practice is not yet expanded in France. It is wished that toad venom doesn't take the relay to existent drugs, but on the opposite, it could be useful to discover other therapeutical interests. 
de nous aider à comprendre et peut-être même un jour à diagnostiquer la sumenue de maladies aussi déroutantes que la schizophrénie ou l'autisme et même qui sait, à les guérir. Diverses publications font état de l'usage de ces sécrétions par ingestion ou par inhalation pour leurs effets psychoactifs aux Etats-Unis et en Australie. Cet usage ne semble pas encore répandu en France. On peut souhaiter que le venin de Bufo ne prenne jamais le relais des drogues existantes et qu'au contraire, on puisse y' découvrir d'autres molécules d'intérêt thérapeutique.

\section{Introduction}

La bufoténine ou 5 hydroxy-N,N-dimethyltryptamine est un alcaloïde indolique isolé de la peau de crapaud pour la première fois en 1893 puis des graines d'une mimosacée, Anadenanthera Peregrina, en 1954. On en trouve également dans l'étoile de mer Asterina Pectinifera, dans les glandes venimeuses de certaines abeilles ainsi que dans divers champignons du genre Amanita. Tout récemment la bufoténine a été mise en évidence dans les sécrétions cutanées de trois espèces de grenouilles du genre Osteocephalus présentes dans le bassin amazonien et la forêt proche de la côte atlantique de l'Amérique du Sud (1). Sa structure chimique est très proche de celle de la psilocybine (5-hydroxy indol au lieu de 4-hydroxy indol), un alcaloïde hallucinogène provenant de champignons du genre Psilocybe et celle du neurotransmetteur sérotonine dont elle est le dérivé diméthylé ( $\mathrm{N}, \mathrm{N}$-diméthylsérotonine). Chez l'homme, ces molécules possèdent de puissantes propriétés psychotropes et sont impliquées dans les désordres mentaux temporaires ou dans des maladies du SNC comme la schizophrénie et autres symptômes psychotiques, probablement du fait de leur point commun tant sur le plan structural que physiologique avec le L.S.D (Fig n ${ }^{\circ}$ 1) vis-à-vis du récepteur 5HT2 (2). Chez les amphibiens, la bufoténine est habituellement associée à un mécanisme de défense vis-à-vis des prédateurs, en raison de ses propriétées nocives (3).

\section{Le venin de crapaud, sour- ce de bufoténine}

Mises à part quelques plantes et champignons du genre Amanita, la principale source d'extraction de la bufoténine est le venin de crapaud. Les crapauds et les grenouilles appartiennent à la classe des amphibiens ou batraciens. Ce sont des vertébrés tétrapodes, poïkilothermes, pentadactiles à la peau nue et humide très riche en formations glandulaires soit isolées (glandes muqueuses), soit en amas (glandes granuleuses). Leur respiration s'effectue au niveau de la peau qui est très

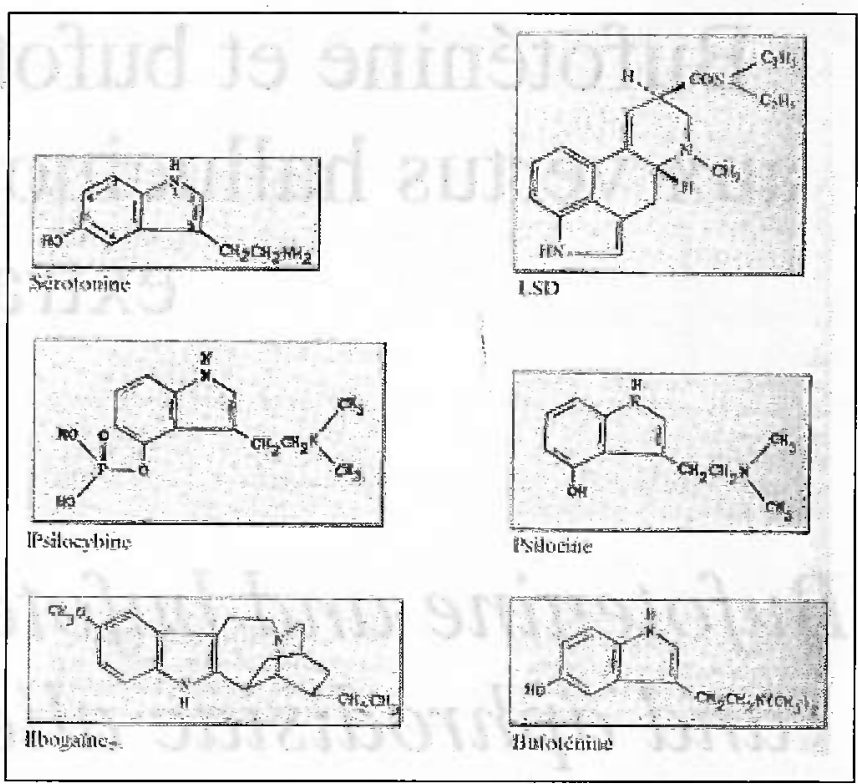

Figure 1 : Structures chimiques de quelques hallucinogènes.

fine (surtout chez les espèces aquatiques), de la muqueuse buccopharyngée, des branchies (chez les larves vivant en milieu aquatique) ou des poumons (chez les adultes). Ils sont anamniotes, pondent dans l'eau et leur développement post-embryonnaire comporte le plus souvent une métamorphose : une partie au moins de leur vie se passe dans le milieu aquatique, en eau douce, puis pour certains, sur un milieu terrestre ayant une atmosphère dont le degré hygrométrique est élevé. Les séjours aquatiques et terrestres varient considérablement d'une espèce à l'autre : certains ne vont à l'eau que pour se reproduire, c'est le cas des crapauds. Les biotopes qui leur conviennent le mieux sont les eaux stagnantes, mares et étangs riches en plantes, fossés ou rivières à courant lent. Les formes terrestres recherchent la terre meuble, les vieilles souches, les failles, les galeries dans le sol. Dans tous les cas, une humidité importante est indispensable. Sous nos latitudes, ils hibernent plusieurs mois : ils se retirent dans les infractuosités du sol ou s'enfoncent dans la vase où ils s'engourdissent (4).

L'ordre qui regroupe les crapauds, les grenouilles et les reinettes (environ 3500 espèces) est celui des anoures, qui tire son nom de l'absence de queue chez les adultes. Il désigne des animaux au tronc raccourci ( 9 vertèbres au maximum alors que les autres amphibiens en comptent 30 à 250), aux pattes bien développées mais de taille inégale (généralement les postérieures sont plus allongées que les antérieures). Les crapauds présentent une répartition mondiale à l'exception de Madagascar, de l'Australie et de quelques îles du Pacifique. Cependant ils ont parfois été introduits dans ces régions (comme le Bufo marinus en Australie à des fins 
insecticides, contre les parasites de la canne à sucre) et s'y retrouvent aujourd'hui couramment et même parfois en surpopulation. Bufo est le genre le plus important et il compte à lui seul environ 250 espèces. Bufo marinus ou crapaud commun des marais, natif de l'Amazone et ses congénères : Bufo vulgaris en Europe, Bufo garganizans en Asie, et Bufo alvarius ou crapaud du Colorado sont les plus connus $(5,6)$. Ils ont une forme lourde, un corps plus ou moins applati et des pattes relativement courtes, peu adaptées au saut. Les doigts sont libres, les orteils plus ou moins palmés, la langue elliptique ou piriforme et libre en arrière : elle peut être projetée en avant:pour capturer des proies (4). Les deux mâchoires sont pourvues de dents. Les téguments sont épais et verruqueux d'où une excellente résistance à la dessiccation ; leur couleur peut changer selon la température. Leur corps est parsemé de pustules qui sont autant de petites glandes venimeuses. De plus derrière les yeux, on note la présence de deux grosses glandes nommées glandes parotoïdes. Ces glandes sont formées de deux types de cellules:

- des cellules à mucus qui sécrètent une substance qui maintient l'humidité de la peau,

- des cellules séreuses qui produisent un « venin » évacué par les pores sur la peau du crapaud et qui ne peut être inoculé faute d'un dispositif adéquat. Il sert cependant de défense contre les prédateurs.

Il faut noter que la terminologie de venin, en ce qui concerne le crapaud, est impropre. En effet, on définit un venin comme une substance toxique, secrétée chez certains animaux par une glande spéciale et qu'ils injectent par piqûre ou morsure. Par conséquent, le crapaud étant totalement dépourvu d'organe inoculateur, il ne lui est pas possible d'injecter sa sécrétion. L'usage ayant entériné le terme de venin, il faut donc toujours sous-entendre dans le cas du crapaud, la sécrétion des glandes parotoïdes (7).

Les glandes parotoïdes produisent plusieurs composés biologiquement actifs comme la sérotonine (5-hydroxy tryptamine ou 5-HT), l'adrénaline, la nor-adrénaline et la dopamine, en quantités variables selon les espèces. Ces glandes produisent également souvent des analogues de ces neurotransmetteurs, principalement de la sérotonine, comprenant la 5-méthoxy-diméthyltryptamine (5-MeO-DMT), la N-triméthyl-5-hydroxy-tryptamine ou (bufoténidine), la dehydrobufoténine (un sel d'ammonium quaternaire de la bufoténine) et la N,Ndiméthylsérotonine ou bufoténine (Fig n²) (5). Les crapauds du genre Bufo excrètent également de leurs glandes parotoïdes des substances appelées bufodiénolides qui comprennent les bufogénines ou bufagines et les bufotoxines qui sont des stéroïdes cardioactifs extrêmement toxiques. Les bufogénines représentent une classe de molécules organiques qui renferment 24 atomes de carbone dans leur structure, qui sont biosynthétisées à partir du cholestérol et qui ne contiennent pas d'azote. Les bufotoxines contiennent par contre de l'azote. Il y a cinq sortes différentes de bufotoxines : la vulgarobufotoxine, la cinobufotoxine, la gambabufotoxine, la marinobufotoxine et l'alvarobufotoxine (5). Comme on l'a dit plus haut, les venins toxiques retrouvés dans les crapauds du genre Bufo dérivent de la tryptamine. La 5-HT et la bufoténine sont présentes chez toutes les espèces de Bufo. Le Bufo alvarius est actuellement la seule espèce connue pour contenir de la 5-MeO-DMT. On trouve de la bufothionine chez Bufo gargarizans (5).

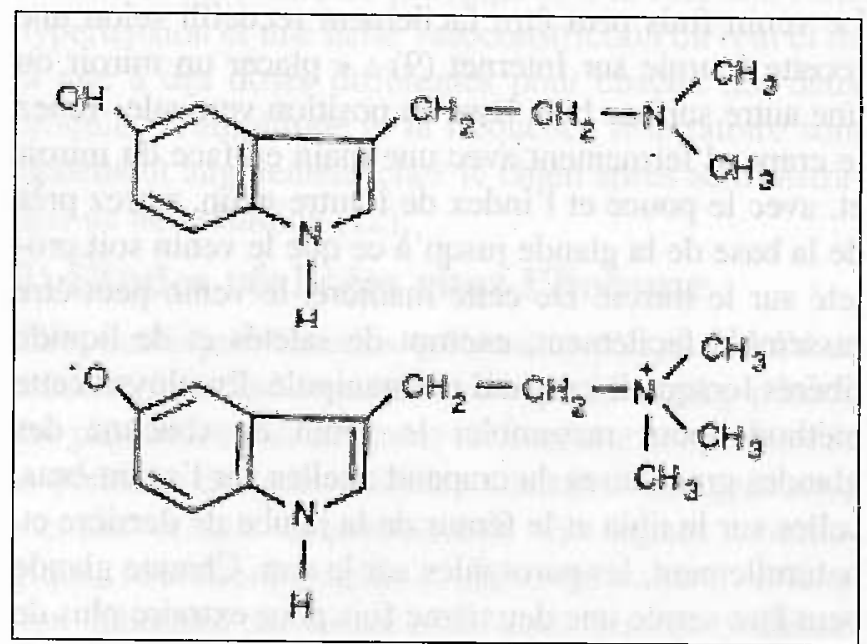

Figure 2 : Structures chimiques de la bufoténine et de la bufoténidine.

La composition et donc la toxicité des sécrétions de Bufo varient considérablement d'une espèce à l'autre mais aussi de la localisation géographique d'une même espèce. Ainsi le taux de mortalité d'animaux non traités, exposés au venin de Bufo marinus varie de la façon suivante : pratiquement $100 \%$ en Floride, très bas au Texas et seulement $5 \%$ à Hawaï. Dix huit espèces différentes de Bufo sont reconnues pour posséder une certaine toxicité (8). Parmi ces espèces, Bufo alvarius semble faire l'unanimité chez les spécialistes en tant que crapaud au venin le plus hallucinogène.

\section{Le Bufo alvarius Giraud ou Crapaud du désert de Sonoran}

Sa distribution couvre surtout le sud-est des Etats-Unis (du Texas à la Californie, particulièrement le désert de Sonoran), et le nord-ouest du Mexique. Ce crapaud 
nocturne se rencontre surtout entre mai et décembre. Son activité est rythmée selon la pluviosité. Il pèse environ $280 \mathrm{~g}$ et mesure en moyenne 10 à $20 \mathrm{~cm}$. Ses coassements durent moins d'une seconde et leur sonorité se rapproche de celle des sifflets des ferry boats. Sa peau, vert olive à marron foncé, tachetée de vert est relativement lisse comparée à celle de Bufo marinus. Ses glandes parotoïdes, au premier abord peu visibles, font environ $2,7 \mathrm{~cm}$ de long. Son venin est de couleur blanche. Il a un aspect crémeux et une odeur très désagréable caractéristique. Comme la plupart des crapauds, il adopte une attitude frustrée lorsqu'il est effrayé, dirigeant ses parotoïdes directement vers l'intrus ; sa prise en bouche par un prédateur peut provoquer, par le biais du venin, la paralysie voire la mort de l'animal.

Le venin frais peut être facilement recueilli selon une recette fournie sur Internet (9) : « placer un miroir ou une autre surface bien lisse en position verticale. Tenez le crapaud fermement avec une main en face du miroir et, avec le pouce et l'index de l'autre main, serrez près de la base de la glande jusqu'à ce que le venin soit projeté sur le miroir. De cette manière, le venin peut être rassemblé facilement, exempt de saletés et de liquide libérés lorsque le crapaud est manipulé. Employez cette méthode pour rassembler le venin de chacune des glandes granulaires du crapaud : celles sur l'avant-bras, celles sur le tibia et le fémur de la jambe de derrière et, naturellement, les parotoïdes sur le cou. Chaque glande peut être serrée une deuxième fois pour extraire plus de venin si vous accordez une heure de repos au crapaud. Quand les glandes sont vides, elles demandent quatre à six semaines pour la régénération. Le venin frais visqueux commence à sécher en quelques minutes et prend la couleur et la texture de la colle à base de caoutchouc. Récupérez le venin sur le miroir, séchez-le complètement, et mettez-le dans un récipient hermétique jusqu'à ce que vous soyez prêt à le fumer (la dose normale pour un adulte de taille moyenne est un morceau de venin sec de la taille d'une tête d'allumette ( 3 à $5 \mathrm{mg}$ )».

\section{Composition de l'extrait parotoïdien de Bufo alva- rius}

L'extrait parotoïdien contient $70 \%$ d'eau, des acides gras, une oxydase, un peu de glutathion $(0,3 \%)$, un peu de stérols $(0,2 \%)$, des protéines et une méthylcarbilamine odorante. II renferme également plusieurs types de substances pharmacologiquement actives :

- des génines cardioactives, représentant $10 \%$ environ du poids de l'extrait sec : ce sont les bufagines ou bufadiénolides qui ont des effets similaires aux hétérosides cardiotoniques des végétaux (digitaline, ouabaïne). Elles ont une structure cyclopenténophénanthrénique. Il s'agit de la bufotaline, de la bufotoxine, de la bufotalinine, de l'hellebrigénine, de la télocinobufagine et de la marinobufagine ;

- des cathécholamines : adrénaline et nor-adrénaline, à propriétés vasopressives et bronchodilatatrices ;

- des indolalkylamines : elles ont une analogie structurale avec l'acide aminé essentiel, le L-tryptophane et ses dérivés physiologiques comme la sérotonine. Il s'agit de la bufothionine, de la déhydrobufoténine, de la bufoténine et de la bufoténidine aux propriétés hallucinogènes ;

- des stérols non cardioactifs : cholestérol, provitamine $D$, gamma-sistérol et ergostérols. Ils ne semblent pas avoir de rôle particulier dans la toxicité du venin ;

- de la tétradotoxine et divers analogues : ce sont des neurotoxines qui bloquent les canaux sodiques des membranes cellulaires.

\section{Légendes et réalités sur l'utilisation du Bufo}

S'il est un animal que l'homme a toujours entouré de mystères, c'est bien le crapaud. Il est l'élément central de nombreux mythes. En Europe comme en Amérique ou en Asie, il est au coeur de multiples croyances à la frontière entre sorcellerie, magie et médecine.

Les apparitions de représentations artistiques du crapaud picturales ou sculpturales sont préhistoriques et le venin de crapaud a sans doute joué un rôle dans les premières religions indo-uraliques de l'âge de pierre. Il était alors utilisé à des fins de chasse collective et comme substance occulte et divinatoire (6). Quelques archéologues pensent que l'homme de Neandertal mangeait déjà des crapauds pour modifier sa conscience. De nombreux squelettes de Bufo ont été retrouvés sur le site olmèque de San Lorenzo (golfe du Mexique). Les sculptures aztèques comportent non seulement beaucoup de représentations de crapauds mais détaillent très souvent les glandes parotoïdes ainsi que les effets qu'elles produisent « dans la tête des hommes ». Il a également été retrouvé des ossements de Bufo dans des vaisselles utilisées lors de pratiques funéraires maya et il est probable que ces restes signifient que les crapauds étaient mis à macérer dans des breuvages à des fins rituelles. Au travers de toute l'Europe, à partir du Moyen Age, on ne compte plus les énoncés de formule où le crapaud est l'un des ingrédients principaux. Celles-ci, le plus souvent des potions destinées à la voie rectale ou percutanée, étaient consommées au cours de rituels de sorcellerie. Il y eut aussi en Angleterre, il y a 
trois siècles des vendeurs d'élixirs miracles et des magiciens qui avalaient des crapauds en public pour prouver l'efficacité de leur remède ou l'étendue de leurs dons surnaturels. Il est difficile d'imaginer la survivance de telles pratiques et pourtant, de nos jours en Haïti, Bufo marinus entre dans Ia composition de drogues destinées au culte vaudou. Au cours des années 70, à Véracruz (Mexique), dans un contexte de rituel de guérison en milieu périurbain, on a observé des curanderos ou brujos (guérisseurs locaux) ayant parmi leur arsenal thérapeutique une pâte fermentée, mélange de parotoïdes de Bufo marinus, de chaux éteinte, de cendres et qui était consommée sous forme de décoction. A la même époque, on trouvait aux EtatsUnis une préparation contenant de la bufoténine, vendue sous le nom de «Stone », « Lovestone », « Blackstone » ou « Rock Hard » qui était disponible dans les épiceries, les magasins de tabac ou auprès des vendeurs de rue. Cette préparation, utilisée comme aphrodisiaque, fut responsable de nombreuses intoxications (8). Des analyses d'échantillons de «Lovestone » ont montré une composition similaire à celle du Ch'an $\mathrm{Su}$ (10). Le « Toad Licking » (consistant à ingérer per os les sécretions des parotoïdes) et le «Toad Smoking » (qui consiste à fumer ces secrétions) sont les termes que les journalistes donnèrent à l'ingestion de venin de Bufo par les utilisateurs de drogues illicites. Cette activité fut pratiquée et l'est toujours dans le but intentionnel d'entrer dans un état de transe à la façon chamanique.

En dehors de ses utilisations à visées occultes ou psychoactives, le venin de crapaud possède un long passé historique en relation avec la médecine. En Chine, au Japon et dans d'autres pays d'Asie du sud-est, les pharmacies traditionnelles Kempo fournissent le Ch'an $\mathrm{Su}$ ou Kyushin, préparé avec du venin de crapaud séché. Depuis plusieurs siècles, les pharmacopées asistiques le reconnaissent entre autre comme un remède des troubles cardiaques en infusion et un aphrodisiaque à appliquer localement. Un produit pharmacologique dérivé des toxines du crapaud fut vendu en France sous le nom de Bufox 2®, comme hémostatique et stimulant des glandes surrénales. Il était commercialisé par les Laboratoires Lepetit et fut disponible jusqu'en 1960 (11).

\section{Pharmacologie de la bufo- ténine et de la bufoténidine}

La bufoténine est la seule des quatre bases azotées à figurer dans le Merck index, sans doute parce qu'elle est inscrite au registre des substances contrôlées aux Etats-Unis, mais actuellement ne figure pas sur la liste des stupéfiants en France.

\section{1) Études expérimentales}

Ces études ont été réalisées sur des préparations d'organes ou sur des animaux vivants. Sur le cœur isolé de grenouille, l'injection de bufoténidine met en évidence une augmentation du tonus puis un ralentissement du rythme, une diminution progressive de l'amplitude des contractions et finalement un arrêt du cœur en diastole:Il semble que la bufoténine ait une action excitatrice périphérique sur les muscles striés et lisses comme l'utérus et l'intestin. Au niveau des vaisseaux, l'action de la bufoténine est de type nicötinique : elle potentialise l'action de l'adrénaline et se révèle faiblement vasoconstrictrice. Elle agit donc de façon indirecte en excitant la sécretion médullo-surrénale. La bufoténine comme la bufoténidine provoquent à la fois une forte hypertension et une nette vasoconstriction du rein et de la rate à des doses différentes pour chacun des deux produits. L'amplitude et la fréquence respiratoire sont également augmentées chez le chien après administration de bufoténidine (12).

\section{2) Études réalisées chez l'homme}

Chez des sujets humains volontaires, l'injection IV de bufoténine à des doses de 0,06 à $0,25 \mathrm{mg} / \mathrm{kg}$ produit des picotements de la tête et du cou, une contraction de la poitrine, des perturbations visuelles, une confusion mentale et une désorientation. Ces signes neurologiques sont accompagnés de signes physiques : coloration aubergine du visage et du cou, nausées, vomissements et nystagmus. Après quelques minutes, on note une relaxation progressive, de la fatigue, un retour lent à l'éveil mental et une euphorie significative durant plusieurs heures. D'autres investigations après administration par voie intra-nasale sous forme de gouttes et par voie IV rapportent, pour des doses élevées ( $1 \mathrm{mg} / \mathrm{kg}$ ) de profonds changements dans la perception visuelle notamment une inversion et une distorsion des couleurs, mais la psychoactivité de la bufoténine demeure assez contestée. Il reste que cet alcaloïde est un puissant vasoconstricteur à haute dose par voie IV ou IM et que le rythme cardiaque étant affecté, une partie du corps est privée d'oxygène, dont le nerf optique, ce qui pourrait être à l'origine de l'apparition des images déformées (13).

Depuis 1987, Andrew T. Weil et Wale Davis, auteurs de l'article «Bufo alvarius, a potent hallucinogen of animal origin » (14) ont interrogé beaucoup d'informateurs dụ sud de l'Arizona qui prétendent avoir fumé sans danger du venin de crapaud et avoir fait l'expérience d'une psychoactivité positive. Personne ne rapporta d'effet toxique. S'étant basés sur ces informations, les auteurs ont émis l'hypothèse que fumer dénaturait sélectivement les composés toxiques. Ils entreprirent donc d'ex- 
périmenter le venin de Bufo alvarius capturé dans la région de Sonoran et conservé dans un flacon hermétique à température ambiante. Une inhalation unique et profonde du venin vaporisé provoqua une psychoactivité en moins de 15 secondes. Tout comme le rapporte Shulgin en 1970 (13), après administration de 5 à $10 \mathrm{mg}$ de bufoténidine en I.V à des sujets sains, l'intoxication fut intense et de courte durée, caractérisée par des hallucinations visuelles et auditives. L'effet puissant se dissipa en 5 minutes, mais les troubles de la perception persistèrent une heure. Aucun effet toxique ne fut ressenti pendant ou après l'expérience et un venin conservé deux ans fut aussi actif que du venin frais (14). On peut remarquer qu'un seul Bufo alvarius secrète 0,25 à $0,5 \mathrm{~g}$ de venin sec. Alors que la concentration en bufoténidine peut atteindre $15 \%$ de ce poids sec, un crapaud peut secréter $75 \mathrm{mg}$ qui, fumés, sont actifs chez l'homme aux doses de 3 à $5 \mathrm{mg}$. En d'autres termes, un seul crapaud procure une quinzaine de doses et un contenant de la taille d'une boîte d'allumettes peut contenir un millier de doses.

\section{Aspects pharmacocinétiques}

La bufoténine comme la bufoténidine sont inactivées par voie orale car une monoamine oxydase présente au niveau de l'estomac, de l'intestin, du foie et du rein détruit le noyau tryptamine et fait disparaître la fonction amine par désamination oxydative. C'est pourquoi ces composés sont généralement sniffés ou rarement injectés.

Cependant, même si elle est sniffée, plusieurs scientifiques affirment que la bufoténine n'est pas du tout psychoactive $(5,6,13)$. La bufoténine est d'ailleurs souvent achetée au rabais comme substance à propriétés psychedéliques, car on prétend qu'elle ne peut pas franchir la barrière hémato-encéphalique en raison de sa faible liposolubilité et de son caractère polaire. Le fait est que la 5-MeO-DMT et la DMT partagent toutes les deux ces critères de bonne liposolubilité et de faible polarité et sont réellement psychoactives. II se pourrait bien qu'une toute petite dose de bufoténine dans le cerveau soit suffisante pour produire des hallucinations. Il a été prouvé effectivement chez le rat, que la bufoténine peut franchir la barrière hémato-encéphalique (15). Elle le fait faiblement mais, comparée aux autres méthyl-indolamines, il y a tout de même une différence. Fuller et al. (15) ont injecté de la bufoténine chez le rat à des doses de 1,30 ou $100 \mathrm{ning} / \mathrm{kg}$ puis ont décapité les animaux à des intervalles de temps variés et étudié la distribution de la bufoténine dans les tissus. Ils ont constaté que les concentrations de bufoténine les plus élevées se retrouvent dans les poumons, le cœur et le sang et les concentrations les plus basses sont dans le cerveau et le foie.
Dans le système nerveux central, les concentrations en bufoténine sont plus élevées dans l'hypothalamus et le tronc cérébral et légèrement plus faibles dans le striatum et le cortex. Les concentrations en acide 5-hydroxy indol acétique (5-HIAA) résultant de la désamination oxydative de la bufoténine sont significativement augmentées dans les quatre zones du cerveau où l'on retrouve la bufoténine. Selon ces auteurs, le fait que le cerveau et le foie soient capables de métaboliser la bufoténine plus rapidement que le poumon et le cœur doit dépendre de la quantité de bufoténine présente.

En ce qui concerne le mécanisme d'action, la bufoténine possède une forte affinité pour plusieurs récepteurs de la sérotonine : 5-HT1D et 5-HT1B. Ces deux récepteurs sont couplés à une protéine $\mathrm{G}$ inhibitrice non liée à l'adénylate cyclase. La bufoténine est également un agoniste efficace des récepteurs 5-HT1D ce qui signifie que son pouvoir agoniste est étroitement lié à son affinité de liaison (-16). Vis à vis du récepteur 5-HT6, la bufoténine est un agoniste plus puissant que la 5-HT mais moins que le L.S.D. Il semble que le groupe hydroxyle en position 5 sur le noyau indole soit important pour l'activité agoniste. Là encore, l'affinité de là bufoténine correspond à sa puissance d'activation (16). Bien que la bufoténine montre une forte affinité pour le récepteur 5-HT2A, elle manque d'activité agoniste détectable. Il est intéressant de noter que la bufoténine ait une grande affinité pour ce récepteur 5-HT2A car des drogues hallucinogènes comme le L.S.D, la présentent également (13). Il est possible que ce trait commun avec d'autres drogues psychédéliques ait une signification.

Des études récentes ont montré la présence d'indolamines méthylées dans le sérum et l'urine de patients psychotiques, ceux plus spécialement atteints de schizophrénie. Les patients normaux ne présentent pas ces substances dans leurs fluides biologiques. Cette découverte suggère donc l'hypothèse d'une synthèse anormale d'agents hallucinogènes méthylés de façon endogène. Dans les années 80 , des méthyltransférases capables de synthétiser des hallucinogènes comme la DMT ou la bufoténidine, ont été décrites chez l'homme au niveau des poumons, du cerveau et du sang mais le produit naturel de cette enzyme reste un mystère. Le groupement donneur de méthyle pourrait être la S.A.M ou S-adénosyl méthionine qui permettrait la méthylation de la tryptamine en DMT, celle de la 5-méthoxytryptamine en bufoténidine et celle de la sérotonine en bufoténine. A la lueur de ces hypothèses et du fait que l'administration de DMT provoque un comportement et des effets comparables à ceux des crises de schizophrénie (hallucinations visuelles et auditives, difficultés à initier les choses ; etc...), il est plaisant de penser 
que ces deux phénomènes pourraient mettre en jeu les mêmes mécanismes (18). Takeda et al.(2) ont ainsi detecté de la bufoténine et de la N-méthyl-5-HT chez presque tous les patients psychiatriques testés incluant des autistes, des épileptiques, des retardés mentaux, des dépressifs, des schizophrènes et des patients atteints de la maladie d'Alzheimer. De même, Kärkkäinen et al.(19) ont constaté une élimination urinaire accrue de la bufoténine chez des personnes agressives suspectées de violences familiales. Il ne s'agit pas nécessairement d'une relation de cause à effet entre la présence de bufoténine et la psychose, mais il y a sûrement une corrélation entre les deux. Ces résultats sont un début fort encourageant, même s'ils ne permettent pas encore d'expliquer les mécanismes de ces pathologies. Il reste en effet à démontrer un « détail » primordial, à savoir si la présence de ces dérivés méthylés, issus du métabolisme anormal de la sérotonine, est ou non la cause des modifications du comportement chez les patients ou la conséquence d'autres anomalies ?

\section{Quelle place pour la bufo- ténine dans la recherche en biochimie médicale ?}

On sait depuis longtemps que la bufoténine et la $\mathrm{N}$ méthyl bufoténine sont présentes chez le genre Bufo au niveau des glandes granuleuses. Ces deux indolamines sont également detectables au niveau du système nerveux central de l'animal. Il en est de même chez Bufo japonicus où les concentrations en alcaloüdes sont particulièrement élevées à la fois au niveau du cerebellum et de la medulla oblongata, ainsi que dans le sang et l'urine de l'animal. Ces observations admettraient donc la possibilité d'un passage des indolamines depuis le SNC vers d'autres compartiments de l'organisme et pourrait expliquer la relation liant le métabolisme de la sérotonine aux désordres psychiatriques humains (20). Ces résultats suggèrent également la possibilité d'utiliser le Bufo japonicus comme modèle pharmacologique pour étudier le métabolisme et la pharmacocinétique de la bufoténine, car s'il a été démontré que la bufoténine exogène ne peut avoir d'effets psychoactifs du fait de son incapacité à pénétrer dans les tissus cérébraux, une production cérébrale in situ de cet alcaloïde pourrait par contre modifier l'état de conscience. Plusieurs substances ont été testées pour faire diminuer le niveau de bufoténine dans le SNC de l'animal. La p-chlorophénylalanine, (inhibiteur de la tryptophane hydroxylase, agoniste partiel du récepteur 5-HT1A) et la ritansérine (āntagoniste du récepteur 5-HT2A) se sont montrées efficaces (21).
Le dosage de la bufoténine urinaire et/ou plasmatique se révélera peut-être un jour un marqueur efficace du diagnostic des désordres psychiatriques et notamment celui de la schizophrénie.

\section{Que dire des propriétés aphrodisiaques de la bufo- ténine ?}

Dans le marché actuel des aphrodisiaques (substances capables d'exciter la libido ou de réveiller l'instinct sexuel), on distingue trois grands groupes de produits : - ceux qui augmentent la libido, c'est-à-dire le désir sexuel : ces aphrodisiaques agissent au niveau du cerveau en modifiant les concentrations de divers neurotransmetteurs ou d'hormones sexuelles spécifiques. Certains d'entre eux peuvent être efficaces chez les deux sexes quoique la plupart agissent sur la concentration en testostérone et de ce fait sont plutôt dédiés aux hommes. Il s'agit de l'ambréine, un constituant majeur de l'ambre grise, une concrétion intestinale sécrétée par le cachalot et également de la bufoténine et de son dérivé méthylé, la 5-MeO-DMT contenues dans les glandes et la peau des crapauds du genre Bufo. C'est l'ingrédient actif du «Love stone » américain ou de la médication chinoise Ch'an su (6). Son utilisation date de l'époque préhistorique et a traversé de nombreuses cultures et de nombreux continents. Son mécanisme d'action n'est pas très clair et aucune propriété anticholinergique n'est décrite pour les sécrétions de la peau de crapaud. Les propriétés aphrodisiaques sont sans doute d'origine centrale comme le sont les autres effets psychoactifs de la drogue mais sa popularité, en ce domaine, reste un mystère (22)

- ceux qui accroissent la puissance, c'est-à-dire l'efficacité de l'érection grâce à l'induction d'une vasodilatation. De telles substances sont surtout utilisées par l'homme bien qu'elles puissent être également efficaces, chez la femme. Le sidénafil (VIAGRA®) ou le tadalafil (CIALIS(B) produisent chez l'homme des érections satisfaisantes et augmentent la satisfaction, sans affecter le désir sexuel. Il en est de même pour la racine de ginseng qui agit comme un antioxydant en augmentant la synthèse de l'oxyde nitreux ainsi que la pression à l'intérieur du corps caverneux, ce qui déclanche la vasodilatation du penis.

- ceux qui augmentent le plaisir sexuel : on y trouve la cantharidine qui fut à l'origine de nombreuses intoxications par le passé, la yohimbine et diverses plantes possédant des effets cardiovasculaires et comportementaux. 


\section{Aspects toxicologiques Contextes d'usages}

Avant que la digitaline ne soit extraite de Digitalis purpura, les peaux de crapauds desséchées et pulvérisées étaient utilisées en médecine populaire comme médication tonicardiaque, expectorante, diurétique ou comme remède contre les maux de dents, la sinusite et les hémorragies digestives. A Taïwan, la soupe de crapaud est encore considérée comme un agent détoxifiant du sang.

Comme nous l'avons déjà dit plus haut, il y a plusieurs types de substances toxiques présentes dans les sécrétions des crapauds : des agents cardioactifs, des catécholamines, des tryptamines et des stérols non cardioactifs. Ces toxines sont localisées dans la peau et les glandes parotoïdes et peuvent s'avérer dangereuses par contact cutané ou oculaire, par ingestion de la peau et des sécrétions ou encore par ingestion de produits commerciaux prétendus aphrodisiaques à base de venin de crapaud.

Les sécrétions de crapaud desséchées peuvent être fumées mais les effets, par cette voie d'exposition sont mal documentés dans la littérature scientifique et médicale.

L'ingestion de l'animal ou l'absorption du venin dermique d'espèces Bufo peut provoquer une intoxication grave de type digitalique avec arhytmies, bloc cardiaque, hypotension et vomissements.

Licht (23) rapporte le cas d'une famille (mère et ses deux enfants) d'un petit village en Amazonie qui fut intoxiquée après avoir mangé des œufs de Bufo marinus.

Un enfant de 5 ans développa un état épileptique après avoir mis dans sa bouche un crapaud Bufo alvarius (24).

On cite le cas d'intoxications accidentelles par projection oculaire de venin de crapand (25).

En France, fin septembre 2004, une poudre brune fut collectée à l'occasion d'une importante fête techno. Elle était vendue 50 euros le $g$ sous l'appellation de « yopo ou cohoba ». L'usager, un homme d'une trentaine d'années la consommait en sniff pour ses effets hallucinogènes. Cette poudre aurait été préparée avec des ingrédients commandés sur Internet. L'analyse toxicologique a mis en évidence la présence de bufoténine et de 5-MeO-DMT : il s'agissait en fait de graines broyées de Anadenanthera peregrina (26).

\section{Signes généraux des intoxications par le venin de crapaud}

Les effets de l'extrait parotoïdien peuvent se manifester de façon locale ou d'une manière plus générale, sur les principales fonctions vitales de l'organisme.

\section{$1^{\circ}$ ) Actions locale au niveau des muqueuses}

Par simple contact avec la peau saine, on note une diminution de la perspiration (anhydrose) mais parfois une irritation cutanée avec érythème, inflammation et douleur (25). En contact avec le nez et la gorge, une douleur et une irritation suivies d'une anesthésie des muqueuses ont été signalées par plusieurs auteurs $(27,28)$. Au niveau de l'œil, la projection du venin ou le contact avec le globe oculaire entraînent un odème fugace, une opacification de la cornée, une hyperhémie conjonctivale et une baisse de la pression intraoculaire. Le simple contact peut conduire à une cécité fugace qui disparaît habituellement en moins de 48 heures $(25,30)$.

\section{$\left.2^{\circ}\right)$ Actions sur les fonctions vitales}

Température : Une hypothermie a été rapportée dans deux cas fatals après ingestion d'un topique aphrodisiaque contenant des bufadiénolides (8) tandis qu'à l'inverse de la fièvre est apparue chez des personnes. ayant consommé des œufs de Bufo marinus (23).

Système cardiovasculaire : l'action est due aux bufadiénolides et est identique à celle de la digitaline (effet inotrope positif) sans doute par un mécanisme similaire d'antagonisme du calcium mais exaltée. A dose modérée, l'extrait parotoïdien renforce, ranime et stimule le fonctionnement du cœur (actions inotrope + , dromotrope + et bathmotrope + ). A forte dose, il excite d'abord puis paralyse l'appareil cardiaque (action chronotrope -) comme la digitaline. Cependant la digitaline provoque une mydriase tandis que le venin produit un myosis. D'une manière très générale, on a pu observer au cours des intoxications un arrêt cardiaque fatal chez un homme de 31 ans ayant ingéré de la soupe de crapaud (31) ainsi que chez un homme qui avait bu un thé à base d'herbes de Chine contaminées par du Ch'an su (9). On note surtout des troubles de la conduction cardiaque : arythmies et/ou bradycardies (32), fibrillations et hypotension $(33,34)$.

Respiration : on observe des dyspnées ou des hyperpnées, parfois une respiration affaiblie pouvant aller jusqu'à l'arrêt respiratoire (8).

Neurologie : un état épileptique s'est développé chez un jeune garçon de 5 ans peu après avoir mis dans sa bouche un Bufo alvarius. Les crises continuèrent durant 1 heure, mais cédèrent sous diazépam et phénobarbital (24).

Systéme digestif : salivation et vomissements, diarrhées peuvent survenir ainsi qu'un engourdissement de la muqueuse orale après ingestion $(24,34)$.

Équilibre électrolytique : une hyperkaliémie $(>6,5 \mathrm{mEq} / \mathrm{l}$ ) similaire à celle observée lors des intoxications digitaliques et une déshydratation ont été rapportées dans trois cas fatals après ingestion d'aphrodisiaque contenant du venin de crapaud (32). 


\section{Traîtement de l'intoxication}

Comme dans le cas général de toute intoxication, il faut prévenir l'absorption en évacuant le toxique, administrer éventuellement un antidote et assurer un traitement symptomatique.

\section{1) Traîtement épurateur}

On procédera de prime abord au lavage des muqueuses cutanées contaminées à grande eau et à un lavage gastrique en cas d'ingestion. La décision de provoquer les vomissements doit être prise uniquement si le patient ne montre ni signe d'irritation des muqueuses, ni signes nerveux centraux. Il est conseillé de ne pas provoquer les vomissements au delà de 30 minutes après l'ingestion du venin. Chez des sujets volontaires et des patients intoxiqués, l'administration d'ipéca très tôt après l'ingestion diminue le taux sérique de la toxine (8), cependant on ne dispose pas d'assez de données pour recommander ou exclure l'utilisation de l'ipéca. On peut également préconiser l'emploi du charbon actif car il adsorbe les glycosides cardiaques et les bufadiénolides et diminue la circulation entéro-hépatique. A pratiquer évidemment chez le patient conscient. Cette thérapeutique s'est révélée très efficace si l'ingestion remonte à moins de 1 heure (35).

\section{2) Traîtements « semi antidotique » et symptomatique.}

Puisque le venin de crapaud contient des génines cardioactives, on a préconisé l'administration de fragments d'immunoglobine antidigoxine (Digidot $\left.{ }^{\circledR}\right)$ chez les patients en hyperkaliémie ou en arythmie. Il faut assurer également une surveillance cardiaque continuelle notamment pour contrôler une bradychardie ou un blocage auriculo-ventriculaire éventuels. Le traîtement de l'hyperkaliémie requiére l'administration de bicarbonates, de glucose et d'insuline. On y associera de l'atropine en cas d'irrégularités cardiaques, de la phénytoïne (DIHYDAN®) pour ses propriétés anticonvulsivantes et antiarhytmiques, et de la lidocaïne, en cas de tachycardie (8).

\section{Diagnostic de l'intoxication}

Le diagnostic repose sur l'examen clinique, une surveillance electrocardiographique, un bilan biochimique classique et electrolytique (dosage de la kaliémie). Des réactions immunochimiques, de type Digoxine sérique (ABBOTT TDx® ou CIBA Corning ACS $®$ ) sont intéressantes à mettre en oeuvre pour confirmer une exposition car les génines cardioactives contenues dans le venin répondent à ces tests mais les concentrations sériques obtenues ne sont pas bien corrélées avec les effets cliniques (8). Un dosage immunologique par polarisation de fluorescence, utilisé normalement pour la détection de la digitoxine a été décrit pour identifier la bufaline dans le sérum humain (36).
Il reste aujourd'hui à développer des méthodes analytiques performantes pour mettre en évidence la bufoténine et la bufoténidine dans les milieux biologiques. Diverses tentatives ont permis d'aborder d'une façon globale le problème dans différents contextes. Ainsi la caractérisation de plusieurs tryptamines dans les secrétions cutanées de la grenouille australienne Litoria aurea, a été réalisée par chromatographie liquide couplée à la spectrométrie de masse (LC-MS) (37). De même une technique par GC/MS a permis d'identifier la bufoténine et divers bufadiénolides dans l'aphrodisiaque « Love Stone » et dans le Ch'an Su (38). Dans les milieux biologiques, on a pu également séparer et doser par LC/MS-MS plusieurs tryptamines dans le cerveau de rats traités par la bufoténine ou la DMT (39). Forsström et al.(40) décrivent une méthode identique permettant la détection de différentes tryptamines dans l'urine chez des patients psychiatriques. Pépin et Duffort (41) ont rappelé récemment les méthodes de détection de la DMT et de plusieurs ß-carbolines dans les milieux biologiques. Il faut à l'évidence reconnaître que les cas médico-légaux d'intoxication par la bufoténine et autres analogues sont très rares actuellement en France et que des techniques d'analyse mieux ciblées et sans doute encore plus performantes se développeront lorsque la demande s'en fera connaître.

\section{Conclusion}

L'histoire et l'utilisation des Bufo ainsi que la tentative de comprendre comment cet animal disgracieux a traversé les siècles exerce toujours une étrange et parfois morbide fascination. Les Etats-Unis comme l'Australie ont été le théâtre, ces trente dernières années de l'avènement d'étranges pratiques le concernant. Il est fumé ou avalé dans le but de s'évaporer dans les brumes de l'hallucination ou comme aphrodisiaque. A l'opposé, les anciennes civilisations amérindiennes, chinoises ou européennes ont largement délaissé cet ingrédient au profit de substances moins repoussantes pour quitter l'état de conscience ou d'une médecine plus rationnelle pour guérir leurs maux. La bufoténine et son effet prétendument psychédélique n'est sans doute pas la seule à être responsable des hallucinations induites par le venin du crapaud si tant est qu'elles existent. Il reste qu'aujourd'hui un engouement pour la consommation du venin de Bufo s'amplifie au mépris des dangers d'une telle pratique ; il atteindrait même les frontières de la France sans doute via les Pays Bas! Mais devons nous vraiment considérer ce phénomène comme un danger ou cela restera-t-il l'apanage de quelques originaux ? 


\section{Références}

1. Costa T.O.G., Morales R.A.V., Brito J.P., Gordo M., Pinto A.C., bloch $\mathrm{Jr}$ C. Occurrence of bufotenin in the Osteocephalus genus. Toxicon. $2005 ; 46: 371-75$.

2. Takeda N., Ikeda R., Ohba K., Kondo M. Bufotenine reconsidered as a diagnostic indicator of psychiatric disorders. Neuroreport. $1995 ; 6(17): 2378-80$.

3. Duellman W. E., Trueb L. Biology of amphibians. McGraw-Hill Book Company ed. 1986 : 228.

4. Brosset F. Bufo bufo : Étude de ses venins et de leurs emplois en thérapeutique. Th. D. Pharm. Lyon. 1989, 89LY01P025, 81p.

5. Little T. Misuse and legend in the «toad licking » phenomenon. Int. J. Addict. $1993 ; 28(3)$ : 521-38.

6. Little T., Goldstein D., Gartz J. Bufo toads and bufotenine : fact and fiction surrounding an alleged psychedelic. J. Psychoactive Drugs. 1996 ;28(3) : 267-90.

7. Poudret D. les constituants toxiques des venins de crapaud et du Ch'an su. Th. D. Pharm. Marseille. 1983: 145p.

8. Poisindex. Toad Toxins. Micromedex® Healthcare Series Vol 126 expires 12/2005.

9. http://mastekush.ovh.org/drogue/toad.htm.

10. Brubacher J.R., Hoffman R.S., Kile T. Toad venom poisoning : failure of a monoclonal digoxin immunoassay to cross-react with the cardioactive steroids. Clin. Toxicol. $1996 ; 34: 529-30$.

11. Limon Duparcmeur N. Bufoténine et bufoténidine : des hallucinogènes issus du crapaud Bufo. Th. D. Pharm.Rennes. 1999, N 8, 98p.

12. Lelogeais P. De l'extrait parotidien du crapaud commun. Ed Foulon, Paris, 1956,195p.

13. McBride M.C. Bufotenine : toward an understanding of possible psychoactive mechanisms. J. Psychoactive Drugs. $2000 ; 32(3): 321-31$.

14. Weil A.T., Davis W. Bufo alvarius : a potent hallucinogen of animal origin. J. Ethnopharmacolgy. $1994 ; 41: 1-8$.

15. Fuller R.W., Snoddy H.D., Perry K.W. Tissue distribution metabolism and effect of bufotenine administered to rats. Neuropharmacology. $1995 ; 34(7): 799-804$.

16. Pauwels P.J., Palmier C., Wurch T., Colpaert F.C, Pharmacology of cloned human 5-HTID receptor-mediated functional responses in stably transfected rat C6-glial cell lines :further evidence differentiating human 5-HT1D and 5HT1B receptors. Arch. Pharmacology. 1996 ; 353 : 144-56.

17. Boess F.G., Monsma Jr F.J., Carolo C., Meyer V., Rudler A., Zwingelstein C., Sleight A.J. Functional and radioligand binding characterization of rat 5-HT6 receptors stably expressed in HEK293 cells. Neuropharmacology. 1997 ; 36(4/5) : 713-20.

18. Ciprian-Ollivier J., Cetkovich-Bakmas M.G. Altered consciousness states and endogenous psychoses : a common molecular pathway ? Schizophr. Research. 1997 ; 28 : 257-65.

19. Kärkkäinen J., Räissänen M., Huttunen M., Kallio E., Naukkarinen H., Virkkunen M. Urinary excretion of bufotenin (N,N-dimethyl-5-hydroxytryptamine) is increased in suspicious violent offenders : A confirmatory study. Psychiatry Res. 1995 ; 58 : 145-52.

20. Takeda N. Serotonin-degradative pathways in the toad (Bufo bufo japonicus) brain : clue to the pharmacological analysis of human psychiatric disorders. Comp. Biochem. Physiol. 1994 ; 107C : 275-81.
21. Takeda N. A phylogenetic analysis of biogenic amines in the central nervous system of various organisms and application to the fields of medical biochemistry. Progress in HPLC-HPCE. $1997 ; 6: 169-211$.

22. Sandroni P. Aphrodisiacs past and present : a historical review. Clin. Autonomic Res. 2001 ; 11 : 303-7.

23. Licht L.E. Unpalatibility and toxicity of toad eggs. Herpetologica. 1968 ; 24 : 93-98.

24. Hitt M., Ettinger D.D. Toad toxicity. N Engl. J. Med. $1986 ; 314: 1517$

25. Van Tittleboom T., Strauven A., Kuhn D. A propos d'un cas d'atteinte oculaire par venin de crapaud. J. Toxicol. Clin. Exp. $1988 ; 8(2): 95-9$.

26. http :www.ofdt.fr/BDD/sintes/ir 0307225 meo dmt.pdf

27. Pantanowitz L., Naude T.W., Leisewitz A. Noxious toads and frogs of South Africa. SAMJ.1998; $88: 1408-14$.

28. Chen K.K., Kovarikova A. Pharmacology and toxicology of toad venom. J. Pharm. Sci. $1967 ; 56: 1535-41$.

29. Ko R.J., Greenwald M.S., Loscutoff S.M. Lethal ingestion of Chinese herbal tea containing Ch'an su. West J. Med. $1996 ; 164: 71-75$.

30. Peyresblanques J. Atteinte oculaire par venin de crapaud. Bull. Soc. Ophtalmol.Fr. 1964 ; 64 : 493-502.

31. Chern M.S., Ray C.Y., Wu D. Biologic intoxication due to digitalis-like substance after ingestion of cooked toad soup. Am. J. Cardiol. $1991 ; 67$ : 443-44.

32. Brubacher J.R., Ravikumar P.R., Hoffman R.S. Analysis of fatal aphrodisiac known as love stone or rock hard. J. Tox. Clin. Tox. $1995 ; 33: 359$.

33. Kwan T., Paiusco A.D., Kohl L. Digitalis toxicity caused by toad venom. Chest. $1992 ; 102: 949-50$.

34. Chi H.T., Hung D.Z.. HU W.H. Prognostic implications of hyperkaliema in toad toxin intoxication. Hum. Exp. Toxicol. $1998 ; 17: 343-46$.

35. Thakore S., Murphy N. The potential role of prehospital administration of activated charcoal. Emerg. Med. J. $2002 ; 19: 63-65$.

36. Dasgupta A., Datta P. rapid detection of cardioactive bufalin toxicity using fluorescence polarization immunoassay for digitoxin. Ther. Drug Monitor. 1998 ; 20 : 104-108.

37. McClean S., Robinson R.C., Shaw C., Smith F. Characterisation and determination of indole alkaloids in frog-skin secretions by electrospray ionisation ion trap mass spectrometry. Rapid Commun. Mass Spectrom. $2002 ; 16: 346-54$.

38. Barry T., Petzinger G., Zito W. GC/MS comparison of the west indian aphrodisiac "Love stone " to the chinese medication " $\mathrm{Ch}$ ' an $\mathrm{Su}$ » : bufotenine and related bufadienolides. J. Forensic. Sci. $1996 ; 41(6)$ : 1068-73.

39. Barker S.A., Littlefield-Chabaud M.A., Connie D. Distribution of the hallucinogens N.N-dimethyltryptamine and 5-methoxy-N,N-dimethyltryptamine in rat brain following intraperitoneal injection : application of a new solidphase extraction LC-APcI-MS-MS-isotope dilution method. J. Chromatog. B 2001 ; 751 : 37-47.

40. Forsström T., Tuominen J., Kärkkäinen J. Determination of potentially hallucinogenic N-demethylated indoleamines in human urine by HPLC/ESI-MS-MS. Scand. J. Clin.Lab. Invest. $2001 ; 61$ : 547-56.

41. Pépin G., Duffort G. Ayahuasca : liane de l'âme, chamanes et soumission chimique. Ann. Toxicol. Anal. 2004 ; $26(1): 76-84$ 